\title{
Quantum computing and the brain: quantum nets, dessins d'enfants and neural networks
}

\author{
Torsten Asselmeyer-Maluga ${ }^{1, *}$ \\ ${ }^{1}$ German Aerospace Center, Rosa-Luxemburg-Str. 2, 10178 Berlin, Germany
}

\begin{abstract}
In this paper, we will discuss a formal link between neural networks and quantum computing. For that purpose we will present a simple model for the description of the neural network by forming sub-graphs of the whole network with the same or a similar state. We will describe the interaction between these areas by closed loops, the feedback loops. The change of the graph is given by the deformations of the loops. This fact can be mathematically formalized by the fundamental group of the graph. Furthermore the neuron has two basic states $|0\rangle$ (ground state) and $|1\rangle$ (excited state). The whole state of an area of neurons is the linear combination of the two basic state with complex coefficients representing the signals (with 3 Parameters: amplitude, frequency and phase) along the neurons. If something changed in this area, we need a transformation which will preserve this general form of a state (mathematically, this transformation must be an element of the group $S L(2 ; \mathbb{C})$ ). The same argumentation must be true for the feedback loops, i.e. a general transformation of states along the feedback loops is an assignment of this loop to an element of the transformation group. Then it can be shown that the set of all signals forms a manifold (character variety) and all properties of the network must be encoded in this manifold. In the paper, we will discuss how to interpret learning and intuition in this model. Using the Morgan-Shalen compactification, the limit for signals with large amplitude can be analyzed by using quasi-Fuchsian groups as represented by dessins d'enfants (graphs to analyze Riemannian surfaces). As shown by Planat and collaborators, these dessins d'enfants are a direct bridge to (topological) quantum computing with permutation groups. The normalization of the signal reduces to the group $S U(2)$ and the whole model to a quantum network. Then we have a direct connection to quantum circuits. This network can be transformed into operations on tensor networks. Formally we will obtain a link between machine learning and Quantum computing.
\end{abstract}

\section{Introduction}

Our brain (which will be seen as a neural network in the following) is known to be a complex system with no chance to describe it at least partly. The reason for this is simple The complexity of a neural network is mainly given by the exponential size of the state space. Interestingly, a quantum circuit has also an exponential state space as usually given by $2^{N}$ where $N$ is the number of qubits. This paper will be discuss the question whether both systems have a similar description. This idea sounds crazy at the first view. But the brain will

\footnotetext{
*e-mail: torsten.asselmeyer-maluga@dlr.de
} 
be viewed as a classical computer with an exponential state space. In the brain, the so-called calculations are given by the signals going through the network. The number of possible ways in a network is exponential large with respect to the size (number of vertices) of the network. A classical computer can be simulate a quantum computer but needs exponential ressources and a far longer calculation time. The motivation for this work is similar idea: is it possible that the brains (or a neural network) can be simulate a quantum computer with smaller ressources?

Here we will discuss this idea. But in the following we don't discuss a direct link between neural networks and quantum computing. Therefore we don't assume things like entangled neurons or similar things. Furthermore we also don't discuss the interaction between machine learning and quantum computing (see $[1,2]$ ). But the main problem of this work is the question: how can we describe such a complex system like the brain?

In physics there were two substantial revolutions: relativity and quantum theory changing our view of nature. In principle, there was also a third revolution which can be called 'Understanding complexity' with topics like deterministic chaos, self-organization, synergy etc. Partly implied by this progress, other branches of natural science like biology and chemistry were developed also much further in particular at the second half of this century. In parallel, mathematics changed also their shape, away from analysis and analytical geometry more in the direction of qualitative methods. Here, the development of topology was central for this direction influencing many areas like algebraic geometry, number and operator theory. Topology can be simply expressed as a qualitative theory of shapes and spaces. So, why not using these ideas to grasp complex systems? This paper used these methods to fill this gap. For that purpose we will concentrate on one of the most complex system, the human brain. Currently there is a big effort to understand it in the Human Brain Project (a EU FET Flagship about 1 billion Euro expensive). But is it possible to understanding processes like learning or intuition without a large simulation of the whole brain? Maybe a combination of advanced physics and mathematics can help. A basic model of neural networks is the famous Ising model. But in the last two or three decades much progress was made to describe spacetime using graphs (the so-called spin nets of Penrose). These graphs are described by quantum mechanics (and are part of quantum gravity). Therefore there is 'room' for changes in this graph by quantum fluctuations. This picture has much in common with the human brain with fluctuations in the neural network (leading to connection changes in the network). So why not use these spin nets to describe the neural network? In this paper we will discuss this possibility by describing a simple model. One guiding principle of this approach is the interaction between connected neurons to obtain a non-trivial model. Usually not only a pair of neurons is involved into an interaction but rather a subset of neurons. We will describe this interaction as feedback loops. Below we will show that this model has features which one would expect (or know) from neural networks. The mathematics behind this approach based on the work of Morgan and Shalen [3] which originally described hyperbolic 3-manifolds (known as Morgan-Shalen moduli space compactification).

In the next section, we will describe the basic model. The brain can be seen as a dynamical graph with electrical signals having amplitude, frequency and phase. Because of the complexity of the graph, it is hopeless to include the whole graph. Instead we form areas of neurons having the same state (ground state or excited state). We describe the interaction between these areas by closed loops, the feedback loops. The change of the graph is given by deformations of the loops. At first view, the interaction between the neurons in the area as represented by loops cannot be neglected. It is equivalent to say that the loops cannot be contracted by deformations. This fact can be mathematically formalized by the fundamental group of the graph. Furthermore the neuron has two basic states $|0\rangle$ (ground state) and $|1\rangle$ (excited state). The whole state of an area of neurons can be obtained fomally by 
suming over all ground and excited states including the corresonding amplitude. Then it is the linear combination of the two basic state with complex coefficients representing the signals (with 3 Parameters: amplitude, frequency and phase) along the neurons. If something changed in this area, we need a transformation which will preserve this general form of a state (mathematically, this transformation must be an element of the group $S L(2 ; \mathbb{C})$ ). The same argumentation must be true for the feedback loops, i.e. a general transformation of states along the feedback loops is an assignment of this loop to an element of the transformation group. Then it can be shown that the set of all signals forms a manifold (character variety) and all properties of the Network must be encoded in this manifold. In the section 3 , we will discuss how to interpret learning and intuition in this model by using the MorganShalen compactification. In particular, the limit for large amplitudes of the signals can be analyzed by using quasi-Fuchsian groups as represented by dessins d'enfants (graphs to analyze Riemannian surfaces). As shown by Planat and collaborators, these dessins d'enfants are a direct bridge to (topological) quantum computing with permutation groups. The structure of the quasi-Fuchsian groups gives a measure of the complexity as shown in section 4 . In the section 5, we will discuss the normalization of the signal seen as reduction to the group $S U(2)$. It reduces the whole model to a quantum network but only for a fixed decomposition of the network into areas. Then we have a direct connection to quantum circuits. Then we can define operations on tensor networks. Formally we will obtain a link between machine learning and Quantum computing.

\section{A simple model of a neural network}

Neural networks as used for deep learning / machine learning are based on the Ising model. Our model is inspired by this model but we will implicitly also use the spin networks known from quantum gravity. It is a difficult task to create a model which is complex enough to generate a realistic behavior but simple enough to work with. For the following, let us assume a graph representing the network of neurons. This graph is non-planar, i.e. the graphs needs a 3-dimensional space to embed them without any intersections of edges. At first we will fix this graph (or network) but later we will allow for fluctuations of the graph (i.e. the change of the edges). Here we are mainly interested in the flow through this network. In the following we will use the words network and graph as synonyms. It is known that:

There is substantial evidence that a "top-down" flow of neural activity (i.e., activity propagating from the frontal cortex to sensory areas) is more predictive of conscious awareness than a "bottom-up" flow of activity.

This fact implies a hierarchical structure inside the network. Then there are areas (part of the network) with a similar activity which are separated from other areas. The whole structure of the network is controlled by another area where this area is connected to many other areas to control them. There are many ways to send a signal from one point in the network to another point. Usually all these ways are used by the signals. The network is very large so that the corresponding area can be seen as a continuous space in good approximation. The whole approach can be seen as a kind of cluster approximation: the network is clustered into areas. Let $\mathcal{N}$ be this network which is decomposed into areas (or sub-networks) $G_{i}$ with

$$
\mathcal{N}=\bigcup_{i} G_{i}
$$

Main idea in the approach is the observation that there is an interaction between neurons, i.e. a signal from one neuron will return directly or after a greater loop. These signals are signals forming a loop which we call feedback loops. This loop can be seen as a real 
interaction between some neurons (or areas of neurons). Usually in an area, there is more than one feedback loop. But the network is not fixed and now we will discuss fluctuations of the network. Usually neurons build new connections and/or remove some other. Because of this behavior some of the feedback loops will be destroyed or new loops will be created. In contrast, there are stable feedback loops which are not destroyed (because they are used very often). This behavior is comparable to contractable and non-contractable loops in topology. Consider a loop in a disk which can be arbitrarily deformed and finally contracted to a point. In contrast, a disk with a hole contains contractable loops (= all loops not going around the loop) and non-contractable loops (= all loops going around the hole). All non-contractable loops are characterized by the number how often the loop winds around the hole. But more importantly, the loop going around the hole is stable with respect to any deformation (or fluctuation) which don't destroy the hole in the disk (or producing a new hole). Therefore we will state: the feedback loops produce the topology of the network.

Closed loops are forming a monoid with concatenation as operation. This monoid can be completed to a group by using the deformation of loops by using the concept of homotopy (one-parameter family of deformations). This group is known as fundamental group $\pi_{1}(\mathcal{N})$ of $\mathcal{N}$, see $[4,5]$ for details. Usually this group is generated by a finite number of generators $w_{1}, \ldots, w_{n}$ forming sequences (the group operation) which are restricted by relations $r_{1}, \ldots, r_{m}$. Then $\pi_{1}(\mathcal{N})$ is given by

$$
\pi_{1}(\mathcal{N})=\left\langle w_{1}, \ldots, w_{n} \mid r_{1}, \ldots, r_{m}\right\rangle
$$

and we will discuss some examples now. The free group $\langle a \mid \emptyset\rangle$ of one generator and no relation are sequences $a^{k}$ for every $k \in \mathbb{Z}$, i.e. $\langle a \mid \emptyset\rangle$ is isomorphic to $\mathbb{Z}$. In a similar manner, the free group $\langle a, b \mid \emptyset\rangle$ is isomorphic $\mathbb{Z} \star \mathbb{Z}$ to a free product. We will later see that this group can be represented by a tree (the Cayley graph of this group). The group $\left\langle a \mid a^{q}\right\rangle$ with one generator and one relation $a^{q}=e$ ( $e$ unit of the group) are sequences $a^{k}$ with $-q<k<q$, i.e. this group is isomorphic to $\mathbb{Z}_{q}$. Other examples are $\left\langle a, b \mid a b a^{-1} b^{-1}\right\rangle=\mathbb{Z} \oplus \mathbb{Z}$ with relation $a b a^{-1} b^{-1}=e$ or $a b=b a$ which is the fundamental group $\pi_{1}\left(T^{2}\right)$ of the torus.

Now we will reverse the argumentation, i.e. for a given group $G$ with finitely many generators we have to consider a space/manifold $X$ so that $G=\pi_{1}(X)$. Using the cellular approximation theorem, the space $X$ must be a 2-complex. This 2-complex consists of 1-cells and 2-cells. The 1-cells give the generators of $G$ and the 2-cells represent the relations in $G$. It is a result of Curtis [6] that any 2-complex is homotopy equivalent to a 2-complex which embeds in $\mathbb{R}^{4}$. This fact is the reason that every finitely generated group can be realized as the fundamental group of a 4-manifold.

The fundamental group $\pi_{1}(\mathcal{N})$ represents the interaction between the neurons and $\mathcal{N}$ denotes now the interaction graph of the neural network. This graph is similar to the neural network, i.e. this graph can be embedded into a 3-dimensional space. Following this argumentation, the 2-complex must be also embedded into a 3-dimensional space or $\pi_{1}(\mathcal{N})$ is the fundamental group of a 3-manifold also denoted as $\mathcal{N}$. By now it is only a conjecture but for the following line of arguments it is unimportant i.e. abstractly we have a 3- or 4-manifold denoted by $\mathcal{N}$ with fundamental group $\pi_{1}(\mathcal{N})$. The choice of this manifold us unique by deep theorems (solution of the Poincare conjecture for topological 3- and 4-manifold by Perelman [7-9] and Freedman [10]).

Furthermore we have a function over this space $\mathcal{N}$ which assigns to every point a state: $|0\rangle$ (ground state) or $|1\rangle$ (excited state). This function is given by the neuron behavior: $|0\rangle$ are signals in $\mathcal{N}$ below the activation of neurons whereas $|1\rangle$ are signals above the threshold. Every signal is given by three parameters: strength (or amplitude), frequency and (relative) phase. These three parameters can be encoded into a complex number $a \in \mathbb{C}$ with state $a|0\rangle$ 
or $a|1\rangle$. For an area $G_{i}$, the state $\left|\phi\left(G_{i}\right)\right\rangle$ is the sum of all $a|0\rangle$ and $b|1\rangle$. To express this fact mathematically, we will assign to every area of neurons a state

$$
|\phi\rangle=a \cdot|0\rangle+b \cdot|1\rangle \quad a, b \in \mathbb{C}
$$

where $a, b$ are complex numbers with a norm $|a|$ representing the strength of the signal for the state $|0\rangle$ in this area and $|b|$ the strength for $|1\rangle$. The phase and the signal frequency of $a$ and $b$ is the relative phase as well the frequency of a signal going through this area. Here, the usage of complex numbers is very important, otherwise we are not able to describe the amplitude, frequency and phase in one structure. The parameter will generate a 2-dimensional complex vector space $\mathbb{C}^{2}$. A parameter change is given by the automorphism of this space as given by the group $S L(2, \mathbb{C})$. If something changed in this area, then we need a transformation which will preserve this general form of a state (mathematically, this transformation must be an element of the group $S L(2, \mathbb{C})$ ). The same argumentation must be true for the feedback loops, i.e. a general transformation of states along the feedback loops is an assignment of this loop to an element of the transformation group (i.e. a homomorphism $\pi_{1}(\mathcal{N}) \rightarrow S L(2, \mathbb{C})$ a representation). Some transformations are ruled out, i.e. if we transform all loops by the same element then we will change nothing (it is a simple gauge of the network).

These ideas can be expressed mathematically which will be done in the following. Main part in our argumentation is the inclusion of neuron interaction by feedback loops. A loop is formally given by loops of the state $|0\rangle$ and of the state $|1\rangle$. A change of the loop by a change of the network or by a change of the currents in the network are given by a map

$$
\pi_{1}(\mathcal{N}) \rightarrow S L(2, \mathbb{C})
$$

which must be preserve the group structure (i.e. it is a homomorphism). Of course, all parameters can be gauged at the same time without any change. This fact can be expressed by an action of the group $S L(2, \mathbb{C})$ on the homomorphism above. This action is given by conjugation (the action $\left.(g, a) \mapsto g \cdot a \cdot g^{-1}\right)$. Mathematically expressed, the set of homomorphism will be denoted by

$$
\operatorname{Hom}\left(\pi_{1}(\mathcal{N}), S L(2, \mathbb{C})\right)=\left\{\pi_{1}(\mathcal{N}) \rightarrow S L(2, \mathbb{C})\right\}
$$

which is unique up to conjugation (the action $\left.(g, a) \mapsto g \cdot a \cdot g^{-1}\right)$

$$
\mathcal{M}=\operatorname{Hom}\left(\pi_{1}(\mathcal{N}), S L(2, \mathbb{C})\right) / S L(2, \mathbb{C})
$$

and finally we will obtain the space $\mathcal{M}$. The transformation group $S L(2, \mathbb{C})$ of our parameters (seen as automorphism of the parameter space as $2 \mathrm{D}$ complex vector space) is well-known in mathematics: it is the isometry group of the 3-dimensional hyperbolic space (honestly it is the universal cover of this isometry group but this difference is unimportant here). Then the space $\mathcal{M}$ is the moduli space of hyperbolic structures on $\mathcal{N}$.

Now let us summarize the model:

1. There is a network of neurons with a hierarchical organization. All signals going through this network

2. There is a set of feedback loops which are stable with respect to a change of the network. These stable loops are identified with non-contractable loops in a 3-dimensional space $\mathcal{N}$. This set of loops has the structure of a group $\pi_{1}(\mathcal{N})$.

3. Every neuron has a state out of two possibilities: $|0\rangle$ (ground state) or $|1\rangle$ (excited state). For an area of neurons we assign a state $|\phi\rangle=a \cdot|0\rangle+b \cdot|1\rangle$ with the complex numbers 
$a, b$. The norm $|a|$ represents the strength of the signal for the state $|0\rangle$ in this area and $|b|$ the strength for $|1\rangle$. The phase and the signal frequency of $a$ and $b$ is the relative phase as well the frequency of a signal going through this area.

4. A transformation of the state $|\phi\rangle$ must be end up with a state again, i.e. the possible transformations forming a group $S L(2, \mathbb{C})$. Then a transformation of feedback loops is a map which assigns every loop (an element of $\pi_{1}(\mathcal{N})$ ) to a transformation (an element of $S L(2, \mathbb{C})$ ) respecting the group structures of the transformation and loops. Gauging of the network is ruled out.

\section{The dynamics of this model: learning and intuition}

The model above is only the frame for a general dynamics. But at first we will ask what we expect if a sensor like the eye gets a signal which will be recognized to be a dog by the neural network? In the previous section we analyzed the structure of the signals inside the network. Here, feedback loops as expression for neuron interactions are the most important ingredients for us. Now let us consider two signals for different objects. One would expect that the endpoints of the signals (after going through the network) are separated from each other. Is this behavior realized in our model? If the network has learned to recognize different objects (where the input signal came from the eye) then the corresponding ways of a signal should have a maximized amplitude and frequency for the signal. Therefore our analysis should be concentrate on the maximized signals inside the networks.

This idea can be realized mathematically by considering a signal function

$$
F: \mathcal{M} \rightarrow \mathbb{C}
$$

with amplitude, frequency and phase over the space $\mathcal{M}$ of all signals as described above. As shown by [3], the space $\mathcal{M}$ can be described by coordinates given by group characters. Let $\rho: \pi_{1}(\mathcal{N}) \rightarrow S L(2, \mathbb{C})$ be one representation. The character is defined by $\chi_{\rho}(\gamma)=\operatorname{Tr}(\rho(\gamma))$ for a $\gamma \in \pi_{1}(\mathcal{N})$. The set of all characters forms an algebraic variety which is equivalent to $\mathcal{M}$. This approach showed that every function over $\mathcal{M}$ must be given by a character or the signal function itself is a coordinate function of $\mathcal{M}$. Of course, this function can be also defined for the areas $\left.F\right|_{G_{i}}: G_{i} \rightarrow \mathbb{C}$. In principle, there are two kind of signals: signals with a convergent amplitude and signals with a divergent amplitude. In our model, both signals have different interpretations. Convergent signals are given for ways which are rarely used (or which do not activate the neurons). In contrast, divergent signals are frequently used ways. As far as we know, the divergent signals are the most important ones because these ways represent the learned signals way. As motivated above, we expect that these ways are separated from other signals i.e. ending at different ends. Expressed differently, the ways of the signal after a learning are forming a tree where the endpoints represent the different recognized objects. This tree must be formed by the signals with divergent amplitudes. Mathematically, we have to understand the compactification of the space $\mathcal{M}$.

Morgan and Shalen [3] studied a compactification of this space or better they determined the structure of the divergent signals. The compactification $\overline{\mathcal{M}}$ is defined as follows: let $C$ be the set of conjugacy classes of $\Gamma=\pi_{1}(\mathcal{N})$, and let $\mathbb{P}(C)=\mathbb{P}\left(\mathbb{R}^{C}\right)$ be the (real) projective space of non-zero, positive functions on $C$. Define the map $\vartheta: \mathcal{M} \rightarrow \mathbb{P}(C)$ by

$$
\vartheta(\rho)=\left\{\log \left(\left|\chi_{\rho}(\gamma)\right|+2\right) \mid \gamma \in C\right\}
$$

and let $\mathcal{M}^{+}$denote the one point compactification of $\mathcal{M}$ with the inclusion map $\iota: \mathcal{M} \rightarrow \mathcal{M}^{+}$. Finally, $\overline{\mathcal{M}}$ is defined to be the closure of the embedded image of $\mathcal{M}$ in $\mathcal{M} \times \mathbb{P}(C)$ by the map 
$\iota \times \vartheta$. It is proved in [3] that $\mathcal{M}$ is compact and that the boundary points consist of projective length functions on $\Gamma$ (see below for the definition). Note that in its definition, $\vartheta(\rho)$ could be replaced by the function $\left\{\ell_{\rho}(\gamma)\right\}_{\gamma \in C}$, where $\ell_{\rho}$ denotes the translation length for the action of $\rho(\gamma)$ on $\mathbb{H}^{3}$ (3D hyperbolic space)

$$
\ell_{\rho}(\gamma)=\inf \left\{\operatorname{dist}_{\mathbb{H}^{3}}(x, \rho(\gamma) x) \mid x \in \mathbb{H}^{3}\right\}
$$

where dist $_{\mathbb{H}^{3}}$ denotes the (standard) distance in the 3D hyperbolic space $\mathbb{H}^{3}$.

Recall that an $\mathbb{R}$-tree is a metric space $\left(T, d_{T}\right)$ such that any two points $x, y \in T$ are connected by a segment $[x, y]$, i.e. a rectifiable arc isometric to a compact (possibly degenerate) interval in $\mathbb{R}$ whose length realizes $d_{T}(x, y)$, and that $[x, y]$ is the unique embedded path from $x$ to $y$. We say that $x \in T$ is an edge point (resp. vertex ) if $T \backslash\{x\}$ has two (resp. more than two) components. A $\Gamma$-tree is an $\mathbb{R}$-tree with an action of $\Gamma$ by isometries, and it is called minimal if there is no proper $\Gamma$-invariant subtree. We say that $\Gamma$ fixes an end of $T$ (or more simply, that $T$ has a fixed end) if there is a ray $R \subset T$ such that for every $\gamma \in \Gamma, \gamma(R) \cap R$ is a subray. Given an $\mathbb{R}$-tree $\left(T, d_{T}\right)$, the associated length function $\ell_{T}: \Gamma \rightarrow \mathbb{R}^{+}$is defined by

$$
\ell_{T}(\gamma)=\inf _{x \in T} d_{T}(x, \gamma x)
$$

If $\ell_{T} \neq 0$, which is equivalent to $\Gamma$ having no fixed point in $T$ (cf. [3, 11], Prop. II.2.15), then the class of $\ell_{T}$ in $\mathbb{P}(C)$ is called a projective length function.

Now we are able to formulate the main result:

If $\rho_{k} \in \mathcal{M}$ is an unbounded sequence, then there exist constants $\lambda_{k} \rightarrow \infty$ (renormalization of the sequence) so that the rescaled length

$$
\frac{1}{\lambda_{k}} \ell_{\rho_{k}}
$$

converge to $\ell_{\rho_{\infty}}$ for $\rho_{\infty}: \Gamma \rightarrow I \operatorname{som}(T)$ a representation of $\Gamma$ in the isometry group of the $\mathbb{R}$-tree $T$, i.e. we have the convergence

$$
\frac{1}{\lambda_{k}} \ell_{\rho_{k}} \Longrightarrow \ell_{T}
$$

But what is the meaning of this result? If the signals in the network are bounded nothing happens. The signals can follow all possible ways and there is no separation of signals. This behavior changes for unbounded signals. According to the result above, the underlying graph for signals degenerates to a tree. But this tree will give the right behavior, it separates the signals. This amazing result can be understood on geometric grounds. The main idea is the usage of the fact that the assignment of a state to the loop by a map $\pi_{1}(\mathcal{N}) \rightarrow S L(2, \mathbb{C})$ is a representative of a hyperbolic structure on the 3-manifold $\mathcal{N}$ which is unique up to gauge (the homomorphism $\pi_{1}(\mathcal{N}) \rightarrow S L(2, \mathbb{C})$ is determined up to conjugation). The set of all signals is given by the space $\mathcal{M}$ with a corresponding function $\mathcal{M} \rightarrow \mathbb{C}$ for the amplitude, frequency and phase. If the value of this function restricted to an area grows up over the time then it is likely that this signal way was learned by the network and it will be stable over time. Interestingly, one has a direct way to visualize this growing: the curvature of the hyperbolic space grows up too. But what is effect on the network. At the vertices, one has the neurons which are connected by the lines. As one can easily see the lines are concavely curved (because of the negative curvature). Then the increasing curvature is given by an increasing curving of the lines. At the limit, the triangle degenerates and become a tree (see Fig. 1 ), see [12]. So, for the maximized signal amplitude (and frequency), the underlying graph of 


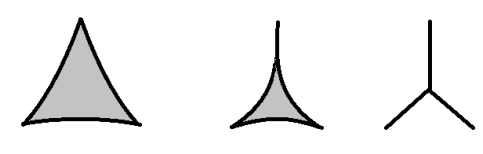

Figure 1. degeneration of a triangle to a tree

the signals degenerates to a tree where the endpoints of the signal are endpoints of the tree (or the leaves of the tree). Here we will point out that the network itself don't degenerates but rather the signals on the network. The signal for low amplitudes and frequencies see the whole graph where the signals for high amplitude and frequency will see a tree. At the end, the network has learned to distinguished between objects. In particular the hyperbolicity of the geometry implies that the distinction is not continuously related to the start point. Consider for example, two objects say 'table' and 'chair' which are not so far away from the recognition of the pattern(three or four legs, some plate, similar size or material etc.). But we learned to distinguished these two objects.

Learning: the separation of the endpoints for the signals in the network by the amplitude of the signal

Above we described a recognition of an object as learned by the network. But how about the process of the learning itself? Here the work of Morgan and Shalen can help to find an inspiration or better an interpretation of learning ion our model again. In the approach above, the number of loops is very important. Usually one needs more then two generators and some relations between the generators to get this result. The relations between the loops can be understood as kind of interaction between the loops (or as global relation). Inspired by this fact, we will understood learning as a two-step dynamics:

1. Increase the number of feedback loops together with relations between them.

2. Increase the amplitudes/frequencies, i.e. repeat the lesson again and again.

Then in the limit, the amplitudes will increase and one will get the separation, i.e. the graph will change to a tree. Abstractly spoken, the learning set is classified into discrete classes where every class corresponds to the endpoint of the tree.

For the process 'learning', the number of loops have to be increased. What happens if we will keep the number of loops fixed but change the relations between the loops? According to the theory of Morgan and Shalen, the tree itself will deform and one will get new endpoints, i.e. the classification of the learning set (used to generate the tree) changes or the network evaluates the input in a new manner. But this process is known as intuition (or creation of an idea)

Intuition: generating new relations between the loops by fixing the loops itself.

Here we will stop for the moment. Our model seems to be a qualitative model at the first view. But the mathematical model behind this approach will also allow for a more quantitative approach. But the main problem is only to fit any realistic data to the parameters of the model (which will imply a lot of work).

\section{On the complexity of network dynamics and dessin d'enfants}

Now we will concentrate on another aspect of this approach: does all endpoints of the tree forming a kind of pattern from which we can extract whether the network has already learned 


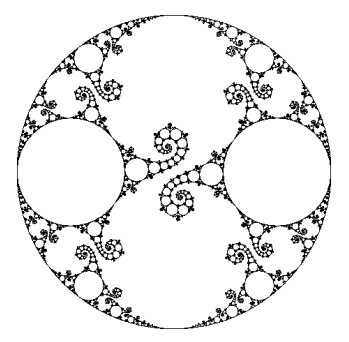

Figure 2. one example of limit (fractal) curves of quasi-Fuchsian groups

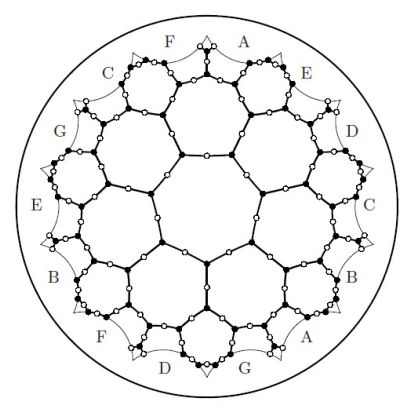

Figure 3. dessin d'enfant for a surface (repainted from [14])

the information? Up to now we don't spoke about the boundary of $\mathcal{N}$, i.e. the area where the signals start and/or end. The boundary allow also for loops $\pi_{1}(\partial \mathcal{N})$ which are embedded into $\mathcal{N}$ with transformations $\pi_{1}(\partial \mathcal{N}) \rightarrow S L(2, \mathbb{C})$. In principle, we are interested in the signals which approach this boundary, i.e. we rather consider the 3-manifold $M=\partial \mathcal{N} \times(0,1)$ together with a representation $\rho_{S}: \pi_{1}(\partial \mathcal{N}) \rightarrow S L(2, \mathbb{C})$. By using this representation, one can define $M$ as factor $\mathbb{H}^{3} / \rho_{S}\left(\pi_{1}(\partial \mathcal{N})\right)$ with metric $d t^{2}+\cosh (t)^{2} g$ with the metric $g$ on $\partial \mathcal{N}$. The manifold $M$ is embedded in $\mathbb{H}^{3}$. In the limit, the process (seen as orbit of a point under the action of $\rho_{S}\left(\pi_{1}(\partial \mathcal{N})\right)$ ) (called quasi-Fuchsian group) will approach the boundary at infinity of $\mathbb{H}^{3}$ given by $\partial_{\infty} \mathbb{H}^{3}=S^{2}$. Then the limit set is the equator of this $S^{2}$ (a Jordan curve which divides the sphere into a northern and southern hemisphere), see [13]. Now there are two possible classes: the process converges regularly, i.e. the limit set is a smooth circle or the process converges to a closed fractal curve. In Fig. 2 we visualizes one examples of this curve for different groups. The appearance of a fractal is a sign of complexity. Indeed, the complexity of the representation $\rho_{S}$ is a direct expression. But this complexity is related to the complexity of the group $\pi_{1}(\mathcal{N})$, i.e. the complexity is given by the interaction in the network. In the other case where the amplitudes are forming a graph (therefore unable to classify information) the endpoints of the graph are not forming a fractal curve. So, the fractal curve is a sign for the completed learning process.

The surface at the boundary $\partial \mathcal{N}$ is given by a tree, see the result of the previous section. It is known that trees are given by polynomials which are locally given at the branching points by $z^{n}$ with $n$ branches. These branching points define locally a ramified covering of the surface over $S^{2}$ (the boundary of $\mathbb{H}^{3}$ at infinity). These data are enough to define a dessin d'enfant [14]. Interestingly, the corresponding dessin d'enfant is given by the triangulation of the surface, see Fig. 3 for an example. But there is also a second line of arguments leading 
to the same result. Let $G^{\prime}$ be a subgroup of the free group $G=\langle a, b \mid \emptyset\rangle$ endowed with a set of relations and $H$ a subgroup of $G$ of index $n$. As shown in $[15,16]$, the permutation representation $P$ associated to the pair $\left(G^{\prime}, H\right)$ is a dessin d'enfant whose edges are encoded by the representative of cosets of $H$ in $G^{\prime}$. The Cayley graph of the free group $G$ is a tree and we have again the relation between the tree at the boundary and dessin d'enfants.

\section{The relation to quantum operations}

Even the analysis of the previous section uncovers a formal analogy between our neural network model and quantum computing. At first we will start with the main assumption: Let $\mathcal{N}$ be the network with a fixed partitioning into areas $G_{i}$ so that $\mathcal{N}=\cup_{i} G_{i}$. For the description of the state, there are two possibilities: one can sum up all states of the areas to get one state for $\mathcal{N}$ but at the same time one will loose many important informations or one forms a tensor state

$$
\left|\Phi_{\mathcal{N}}\right\rangle=\left|\phi_{G_{1}}\right\rangle \otimes\left|\phi_{G_{1}}\right\rangle \otimes \cdots \otimes\left|\phi_{G_{i}}\right\rangle \otimes \cdots
$$

to preserve this information. Furthermore this state reflects also the exponential size of neural network states in general.

Now it is possible to define operations on this state. For instance, if the signal amplitudes increases so that all neurons in the area will change from the ground state $|0\rangle$ to the excited state $|1\rangle$ then this change can be seen as NOT gate (in the quantum computing)

$$
N O T=\left(\begin{array}{ll}
0 & 1 \\
1 & 0
\end{array}\right)
$$

acting on the the state $\left|\phi_{G}\right\rangle$ of the area. In principle, one can define every 1-quibit gate in the quantum computing. Of course, the corresponding state is not a quantum state because the state is not normalized. But it is an easy task to normalize the state $\left|\phi_{G}\right\rangle=a|0\rangle+b|1\rangle$ for every area by dividing the coefficients by the expression $\sqrt{|a|^{2}+|b|^{2}}$. Then every element of $S U(2)$ will preserve this state. A similar argumentation can be used to introduce 2-qubit operations. Here one needs an interaction (or coupling) between the two areas, say $G_{1}$ and $G_{2}$, with a common state $\left|\phi_{G_{1}}\right\rangle \otimes\left|\phi_{G_{2}}\right\rangle$. Now one can choose the coupling so that the excited state $|1\rangle$ of $\left|\phi_{G_{1}}\right\rangle$ activates the neurons in $G_{2}$ which is nothing as the action of the NOT gate on $\left|\phi_{G_{2}}\right\rangle$. This scenario can be described as the action of the CNOT gate on $\left|\phi_{G_{1}}\right\rangle \otimes\left|\phi_{G_{2}}\right\rangle$. It is certainly possible to construct more operations but in principle the CNOT and the 1-quibit operations are enough to represent quantum circuits [17-19].

This description showed that our model of a neural network has much in common with quantum circuits. We don't have in mind that a neural network and a quantum computer are the same. Here we want to discuss only the similarities between both descriptions by using our model.

\section{Conclusion}

In this paper we described a qualitative model for neural networks like the human brain. Main part of this paper is a new model to understand the behavior of neural networks. The main idea is a subdivision of the network into areas with a state given by the signal through this area consisting of a sum of a ground state $|0\rangle$ and excited state $|1\rangle$. The interaction between neurons was described by loops (feedback loops) i.e. by an signal exchange between the neurons. The state space of all signals was analyzed to get an exceptional behavior for signals with a large amplitude. These signals forming a tree which can be used to understand 
the process of learning in a neural networks. Furthermore, the signals at the endpoints of this neural network are related to fractal structures (quasi-Fuchsian groups).

By analyzing the state space in detail, we are able to get a formal relation to quantum circuits and quantum computing. This relation is purely formal and has nothing to do with features like entangled neurons etc. The mathematical description of our model and of quantum circuits have some aspects in common which was described in the previous section.

From the philosophical point of view, this approach has global features which controls the behavior of sub-components. So, the interaction between neurons given by the feedback loops controls the behavior of the neurons in the loop. In philosophy, one calls this behavior top-down causation:

Top-down causation refers to the effects on components of organized systems that cannot be fully analyzed in terms of component-level behavior but instead requires reference to the higher-level system itself.

This model serves as a simple example of this principle. But it showed that a qualitative model can be more successful then a large scale simulation.

\section{References}

[1] V. Dunjko, J. Taylor, H. Briegel, Phys. Rev. Lett. 117, 130501 (2016)

[2] H. Briegel, V. Dunjko (2017), arXiv: 1709.02779

[3] J. Morgan, P. Shalen, Ann. Math. 120, 401 (1984)

[4] M.J. Greenberg, J.R. Harper, Algebraic Topology (Addison-Wesley, New York, 1981)

[5] M. Nakahara, Geometry, Topology and Physics (Adam Hilger, Bristol and New York, 1989)

[6] M. Curtis, On 2-Complexes in 4-Space, in Topology of 3-Manifolds and Related Topics, edited by M. Ford Jr. (Prentice-Hall, Englewood Cliffs, N.J., 1962), pp. 204-207

[7] G. Perelman (2002), arXiv:math.DG/0211159

[8] G. Perelman (2003), arXiv:math.DG/0303109

[9] G. Perelman (2003), arXiv:math.DG/0307245

[10] M. Freedman, J. Diff. Geom. 17, 357 (1982)

[11] J. Morgan, P. Shalen, Ann. Math. 127, 403 (1988)

[12] J. Morgan, P. Shalen, Ann. Math. 127, 457 (1988)

[13] S. Moroianu, J.M. Schlenker, J. Diff. Geom. 83, 75 (2009)

[14] E. Girondo, G. Gonzalez-Diez, Introduction to Compact Riemann Surfaces and Dessins D'Enfants, Vol. 79 of London Math. Soc. Students Texts (Cambridge University Press, New York, 2012)

[15] M. Planat, H. Zainuddin, Mathematics 5, 1 (2017), arXiv: 1601.04865, doi:10.3390/math5010006

[16] M. Planat, Rukhsan-Ul-Haq, Adv. in Math. Phys. 2017, 5287862 (9 pages) (2017), arXiv: 1701.06443

[17] D.P. DiVincenzo, J. Smolin, Physical Review A 51, 1015 (1995)

[18] D.P. DiVincenzo, Physical Review A 51, 1015 (1995)

[19] D.P. DiVincenzo, Proceedings of the Royal Society London A (1996) 